www.conferenceie.ase.ro

\title{
ACCESS CONTROL IN SS-BI SYSTEMS
}

\author{
Aida-Maria POPA \\ Bucharest University of Economic Studies, Romania \\ aida_popa@yahoo.com
}

\begin{abstract}
Currently, SS-BI (Self-Service Business Intelligent) targeting is a way for final users to get rid of dependence on IT departments and gain some independence in how data is processing and how visualization works. Today, SS-BI is one of the most popular trends for companies that want to streamline their business with modern data processing technologies. The end user has the ability to design and deploy their own objects (reports, queries, analyzes, dashboards, etc.) without any intervention from the IT department and without any basic model or architecture. Bringing SS-BI into a business implies a great challenge for analysts, but the benefits are easy to note. Bringing data usage to lower levels of leadership gives users the ability to engage more actively in the company and understand the entire business they run their day-to-day activity. But also, an important aspect is the control of data access, viewing and data manipulation by users. Independence in this case may be limited. It is expected that when a user builds an object (for example, a report), they can give viewing or manipulation rights to other users, but, for this action, a superior (manager, administrator) has to authorize the entire process. In this context, the pyramidal access control model based on roles and attributes with validation appears.
\end{abstract}

Keywords: access control, pyramidal access control model, Self-Service Business Intelligent, user rights

JEL classification: D83

DOI: $10.12948 / \mathrm{ie} 2019.03 .07$

\section{Introduction}

Under the conditions of developing interest and technologies relevant to the Business Intelligence concept, companies must keep up with and make the right choices for their business. Implementing new and expensive technologies but not fitting with the business environment will obviously not provide the desired benefits and, also represent a waste of financial and human resources.

In 2019, according to statistics, the most approached Business Intelligence concepts are data quality / data management (master data management), data / reports visualization and selfservice Business Intelligence. On the opposite side of the ranking are BI cloud, Visual design standard and IoT analytics.

At the core of any SS-BI project are the strategic objectives and objectives of the organization. Data and information in the company are useful when they contribute to improving business efficiency and improving performance by supporting strategic decision-making [1]. However, the complete ignorance about access and security of data and information can, on the contrary, drive the whole business to stranded major losses.

The main role of the Business Intelligence systems is to provide accurate and detailed information to the management of the company in order to support the business activity through a performant decision-making process. Based on the current economic market trends to be more dynamic, it is important for a company to use both technological and human resources to develop effective plans for developing the business activity. In this case, the power of Self- 
www.conferenceie.ase.ro

Service Business Intelligence systems is proven, by giving the power to the lower level users to be closer to data and information about the activity of the department, or more, about the entire company activity (investments, profits, people performance, sales, etc.) [2].

\section{Some aspects regarding access control and SS-BI systems}

SS-BI is a comprehensive concept that brings together both the software component (the BI application) and the entire information system of the company along with the human resource that is part of it. Creating an end-user independent of the IT department was the starting point for developing SS-BI systems [3]. The four aspects that outline the advantages of using SS-BI systems are: integration, sharing, analysis, and visualization of objects. These bring some notable advantages compared to traditional Business Intelligence systems (Figure 1).

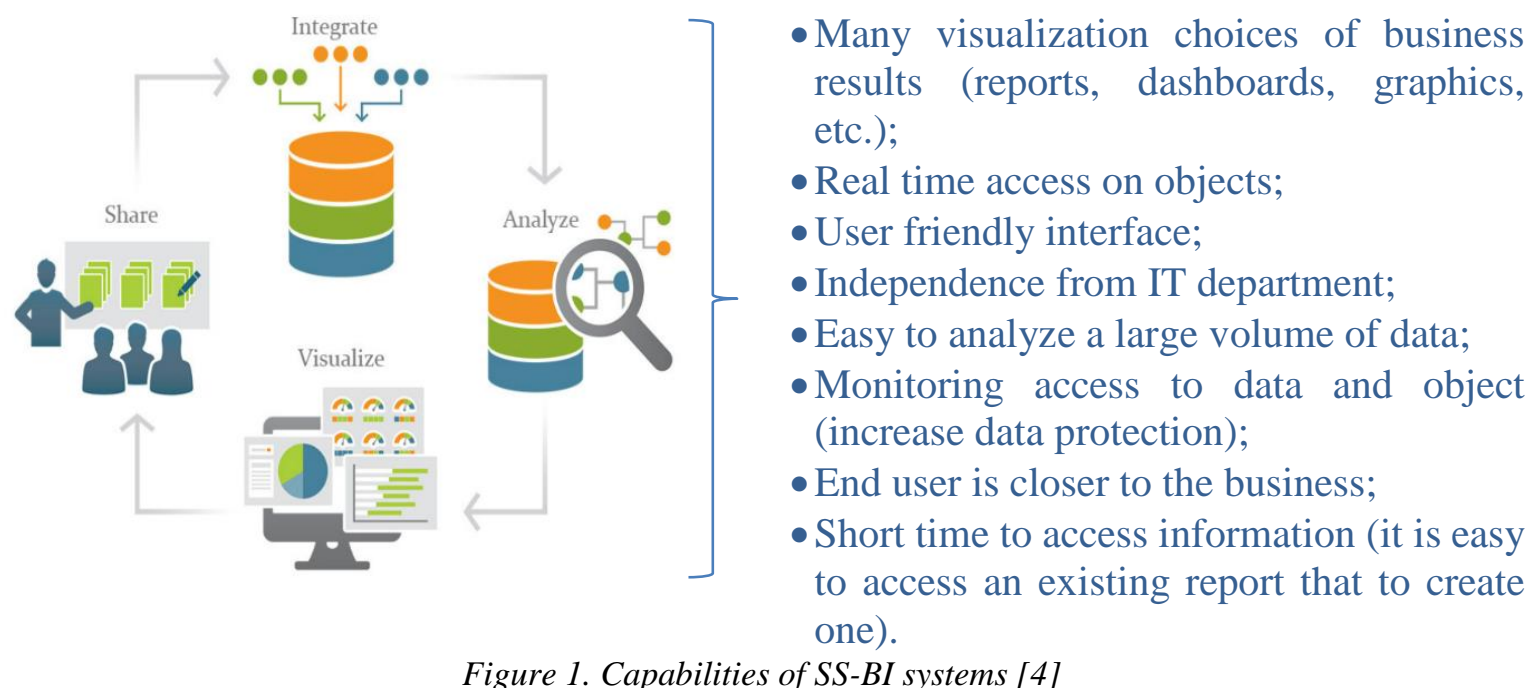

In general, access control defines a security mechanism for accessing various components of a software application [5]:

a) effective access to the application;

b) access to data and information viewing;

c) access to data and information handling (addition, modification, deletion);

d) access to manipulation of end-user rights on data and information.

\section{Pyramidal access control model}

Based on the many other studies related to the access control models, the paper wants to present another access control model consisting of three levels: base objects level, data level and user rights and validation level. The top level represents the particularity of the model because allow user to have access to real and accurate data and information. If one created object doesn't contain valid data and information, this object will be no longer available for users because this can create confusion and can lead end users to do inappropriate business decision [6].

\subsection{Use of access control in SS-BI systems}

The importance of access control in SS-BI systems can be easily discovered: empower one user with rights over objects and data sources can bring both advantages and disadvantages. The main role of access control is to increase the number of advantages and decrease as much as it can the possible disadvantages. 
The control access component needs to have two important parts:

1) Access control validation

2) Access control settings

Access control validation refers the software component that is integrated in the SS-BI system and check using specific algorithms if one user can do some action (create a new object, view an existing object, etc.).

Access control settings refers all the rights, roles, attribute that users, objects and data sources can have.

Schema of access control components inside the SS-BI systems is represented in Figure 2.

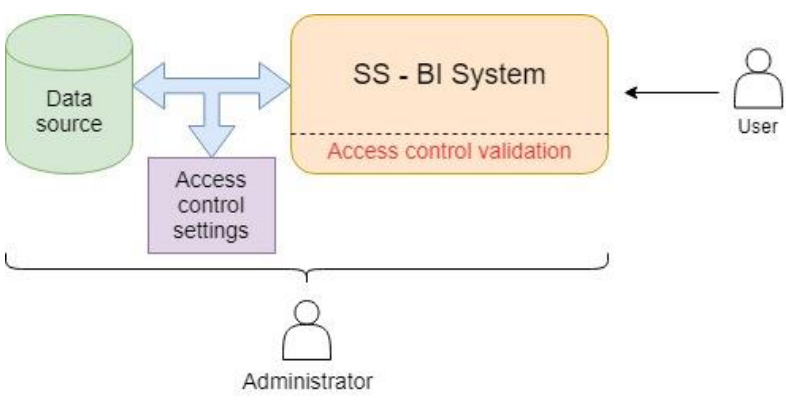

Figure 2. Schema of access control in SS-BI systems

\subsection{Specific concepts used in the definition of the pyramidal access control model}

The user who create an object became user-admin for that object. That means he has all the rights to view, modify or delete the object. Also, if his object is valid and the user-admin get permission to have control of the other user`s rights on that objects, he can give access rights to many other users (the list of users that can pe empowered to have rights on object is given from the administrator/manager).

Access control database is a relational database used to manage the rights, roles and attributes. This database contains one table with the list of users, one table with a list of data sources, a list of objects and a lot of list with permissions, rights and relations between those.

\subsection{Architecture of the pyramidal access control model}

The Figure 3 shows a structured model of access control based on three levels of rights and control: rights over created objects, rights over data sources and control rights over objects and data sources (including validation of objects).

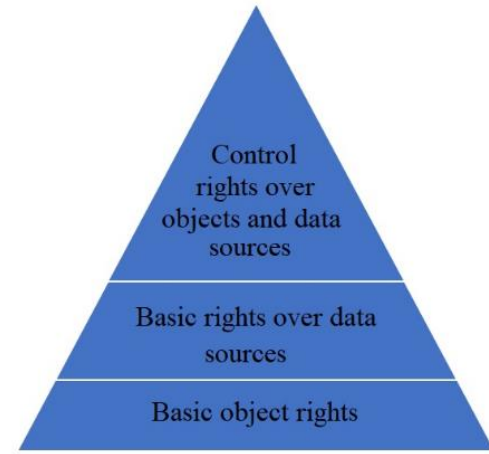

Figure 3. Architecture of the pyramidal access control model 
1) Level 1 - Level of Object Rights. Objects include reports, dashboards, export formats, analytics, graphs, etc. generated on the base of data sources. The basic rights to objects are viewing, adding, modifying, and deleting. All these rights are automatically assigned to the user who created the object (user-admin of the object). Administrator or user-admin (if it has control rights) can give other users, total or partial rights to the object.

2) Level 2 - Level of rights over data sources. The basic rights on the data sources are those of viewing, adding, modifying and deleting. A user with rights to data sources also automatically has rights to objects created based on data sources, but only similar rights (if the user is only viewing on a data source, he will only have the right to view the object, without adding, modifying or deleting the object).

3) Level 3 - Level of control of rights over objects and data sources. This level is called "control" because it has two main functions: the management of user access to objects and data sources and the control of access to valid and real sources. The user-admin can have the administrator rights to share an object with a specific list of users only if he validates the object. This allows inaccurate, incomplete or irrelevant analyzes to reach other users who can use them to make decisions in the company's business. Security is not just about protecting the information, but it is also about correctness. It's simple to take as a basic example: a report created by a user of a department, that has as a result a much higher profit than the real company profit it really is for the current year, will cause another user from another department to allocate larger funds for new investment based on the delivery results of the initial user. This is obviously causing losses to the company, and none of the users can really be blamed for the decision.

\subsection{Steps of creating and implementing the pyramidal access control model in SS-BI systems}

The Figure 4 shows all the steps necessary to create and implement the pyramidal access control model in SS-BI systems. This model has the most important steps that are used in any project and, skipping one of the steps or changing the order can lead to an unsuccessful implementation.

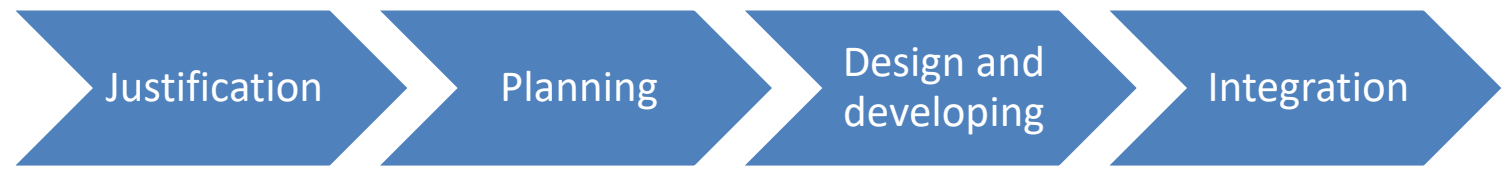

Figure 4. Architecture of the pyramidal access control model

1) Justification

- business evaluation

- analysis of the current SS-BI system

- defining administrator requirements

2) Planning

- defining types of users

- defining types of rights (view, add, change, delete)

- defining types of roles

- defining attribute types

3) Designing and developing

- designing the control database based on the elements defined in the planning stage

- designing and developing the access control interface

4) Integration 
- integrating the control database as a connection between the SS-BI system and the data sources

- integrating the control software application into the SS-BI system

There are many areas of activity where access control functionalities are not negotiable (financial services, medical and pharmaceutical, government systems, etc.). Choosing the best administrator to manage de access control database is basically the first important step that needs to be taken seriously because of him the business can become very performant or can bring loses that are hardly to recover. If the platform that is used doesn't have all the necessary components, those will need to be cover with more human resources, more processes and more costs in technology [7].

\subsection{The necessity, advantages and disadvantages of using pyramidal access control model inside SS-BI systems}

The most important thing that pyramidal access control model brings in SS-BI system is the control of created objects. A proper validation is the main purpose of this model. There are a lot of advantages, common to many other models like:

- stored objects are complete and consistent;

- storing a large volume of objects can bring more information;

- quick access to information (it is easy to access an existing object than to create one);

- performant management of users, rights and roles;

- performant management of data sources and objects (information);

- reduce data analysis time (for complex objects);

- empower users with access rights can bring them closer to the activity of the company.

Also, it is important to mention some disadvantages that this model can have. Empowering an end user with permanent rights to modify or delete objects can became a tricky thing to do. The next important step in development of the proposed model should be to find a proper way to manage also the access time. A temporal access can be really useful and could give control to anything that can happen with an object. Another disadvantage can be the fact that the object table will have sometimes duplicate objects. If one user creates a report but he doesn't give permissions to another user that need the same report, the second user will create practically another object that is similar with the first one. In this case, it is very important to create a good management of objects rights in order to avoid an unnecessary loading of the database.

\section{Conclusions}

In conclusion, pyramidal access control model is an advanced model because doesn 't manage just the rights over data and information, but also ensure a real and accurate image on business activity. The main purpose of the model it is to catch together two important aspects related to Self-Service Business Intelligence systems: access control to data and access to valid data. Based on the fact that one of the most advantages of SS-BI systems is the sharing capabilities, working with accurate information is a priority. Confusing or invalid information lead managers to make erroneous decisions that may affect the long-term business activity of the company.

\section{References}

[1] M. Velicanu, Gh. Matei, Tehnologia inteligenta afacerii, ASE Publishing House, Bucharest, 2010, ISBN 978-606-505-311-3. 
www.conferenceie.ase.ro

[2] A.M. Popa, "Data warehouse pyramidal schema architecture - Support for business intelligence systems", in Proceedings of the IE 2015 International Conference, Bucharest, Romania, 2015.

[3] "Self-Service BI - An Overview". [Online]. Available: https://bi-survey.com/self-servicebi. [Accessed March, 2019].

[4] "History of Business Intelligence to Be Evolved from Then \& Now". [Online]. Available: https://roosboard.com/blog/history-of-business-intelligence-to-be-evolved-from-then-andnow.html. [Accessed March, 2019].

[5] Qi Hui, Di Xiaoqiang, Li Jinqing, "Formal definition and analysis of access control model based on role and attribute", Journal of Information Security and Applications, vol. 43, December 2018, pp. 53-60.

[6] M. Mammass, "An Access Control Model based on the concepts of Organization and Service for Large Infrastructures", Second International Conference on Intelligent Computing in Data Sciences (ICDS 2018), Procedia Computer Science 148 (2019), pp. 571-579.

[7] J. Underwood, "Self-Service BI Governance and Security Risks". [Online]. Available: https://www.jenunderwood.com/2016/12/12/self-service-bi-governance-security-risks/, December 2016. [Accessed March, 2019]. 